\title{
Enhanced Functions for a Parallel Multicore Ground Proximity Warning System
}

\author{
David Mueller \\ Institute of Flight Systems \\ German Aerospace Center (DLR) \\ Braunschweig, Germany \\ david.mueller@dlr.de
}

\author{
Umut Durak \\ Institute of Flight Systems \\ German Aerospace Center (DLR) \\ Braunschweig, Germany \\ umut.durak@dlr.de
}

\begin{abstract}
The German Aerospace Center (DLR) is currently developing a Terrain Awareness and Warning System (TAWS) utilizing the worst case execution time (WCET)-Aware PaRallelization of Model-Based Applications for HeteroGeneOus Parallel Systems (ARGO) approach. The basic functions of the TAWS were modelled based on the Enhanced Ground Proximity Warning System (EGPWS) by Honeywell which is installed in DLR's research aircraft ATRA. Moreover, beyond the existing capabilities, "Enhanced" functions are being implemented in order to demonstrate the possibilities that become available through the use of parallelization for multicore platforms and to investigate new approaches to improve the protection against Controlled Flight into Terrain (CFIT) accidents. After introducing the parallel multicore TAWS development, this paper describes the enhanced functions that make use of the Electronic Flight Instrument System (EFIS) for displaying a predictor symbol that represents the aircraft's future position as well as possible terrain conflicts.
\end{abstract}

Keywords-TAWS, CFIT, multi-core, parallelization

\section{INTRODUCTION}

Parallelization of model-based applications provides efficient use of multicore platforms and thus increases the information processing capabilities, so that it furnishes effective software development for complex and feature-rich real-time systems. Parallelization and multicore platforms for airborne systems have been studied in the last decade. However, current literature mostly focuses on the applicability of multicore platforms regarding safety constraints $[1,2,3,4$, 5]. DLR's effort instead aims at investigating the benefits of parallelization to create next generation avionic systems.

In this context, this endeavor investigates replacing the commercial Enhanced Ground Proximity Warning System (EGPWS) alerting envelopes which trigger the look-ahead warnings by continuous flight simulations executed at runtime. This way, the aircraft's future position can be precisely identified, checked for terrain conflicts and presented in the Navigation Display (ND) in the form of a predictor symbol. Furthermore, in the case of a detected conflict, said simulations are used to find safe alternative flight paths that are displayed in the ND as well. The pilot can then decide which action to take in order to clear the conflict by steering the predictor symbol onto one of the displayed trajectories. Further support is offered by cues displayed in the Primary Flight Display (PFD), showing the minimum roll and/or pitch angle required for an evasive action.
This paper first presents the ARGO project before introducing the development of a parallel multicore Terrain Awareness and Warning System (TAWS). After elaborating on the conventional and enhanced features, the conceptual design of the predictor symbology is explained.

\section{THE ARGO PROJECT}

While the increased computing power and other performance benefits associated with parallel architectures are very promising for a variety of applications, developing embedded parallel real-time software for multicore processors is still a time-consuming and error-prone task. Some of the main reasons are:

- It is hard to predict the performance of a parallel program and therefore hard to determine if real-time timing constraints are met.

- New potential errors like race conditions and dead locks are introduced. These errors are often hard to reproduce and therefore hard to test for.

- The parallelization approach of an application is optimized for a specific number of cores resulting in a high porting effort when there is need for changing the number of cores.

In order to address these challenges, the EU project ARGO, which is funded by the Horizon 2020 Research and Innovation Program, was created. It is addressing the code generation for WCET aware parallelization of model-based applications for multicore systems by providing an integrated toolchain.

The ARGO model-based design workflow (Fig. 1) starts with the controller modeling in which the application models are implemented using the Scilab/Xcos open source model-based design and simulation environment [6]. The workflow includes target architecture specification using an Architecture Description Language (ADL), model-to-text transformations for code generation and a series of code (text-to-text) transformations, based on the information about task dependencies in terms of data needed to be communicated between tasks as well as shared resources access characteristics. The core and system level WCET step calculates the multicore worst case execution time for the target architecture. With iterative optimizations, an explicit parallel program representation with synchronizations and address 


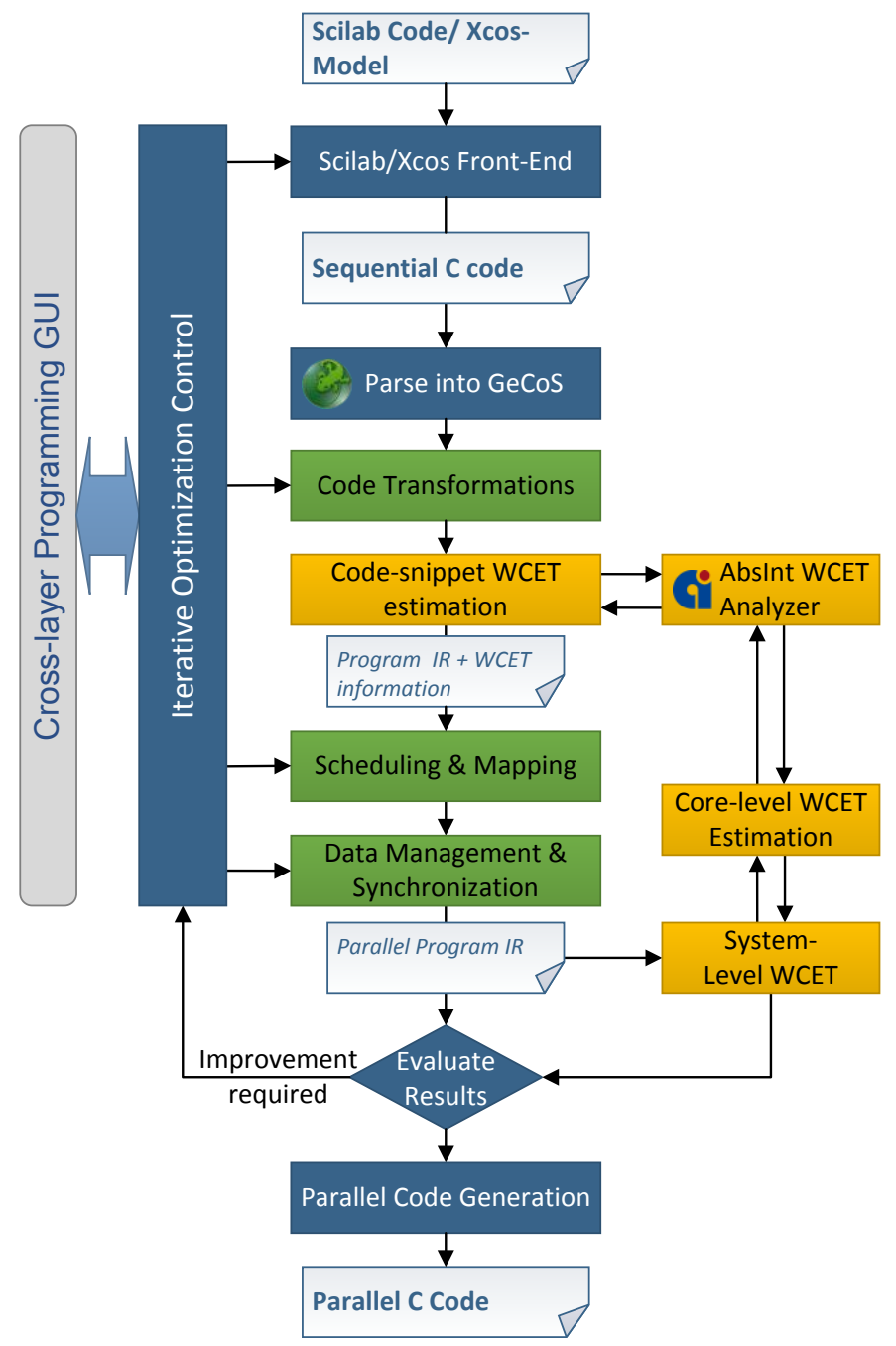

Fig. 1 The ARGO model-based design workflow with integrated toolchain.

mappings is reached at the end. The details of the proposed approach can be found in [7].

A TAWS was chosen as DLR's use case contribution to the ARGO project. "TAWS" defines a set of features which aim to prevent Controlled Flight Into Terrain (CFIT). This type of accident was responsible for many fatalities in civil aviation until the Federal Aviation Administration (FAA) made it mandatory for all turbine-powered passenger aircraft with six or more passenger seats registered in the U.S. to have TAWS equipment installed [8]. There are various TAWS options available in the market for various platforms in various configurations. The core feature of such a system is creating visual and aural warnings between $30 \mathrm{ft}$ to $2450 \mathrm{ft}$ Above Ground Level (AGL) in order to avoid controlled flight into the terrain. These warnings are categorized in five modes, which will be referred to as "basic modes" or simply "GPWS" in this paper:

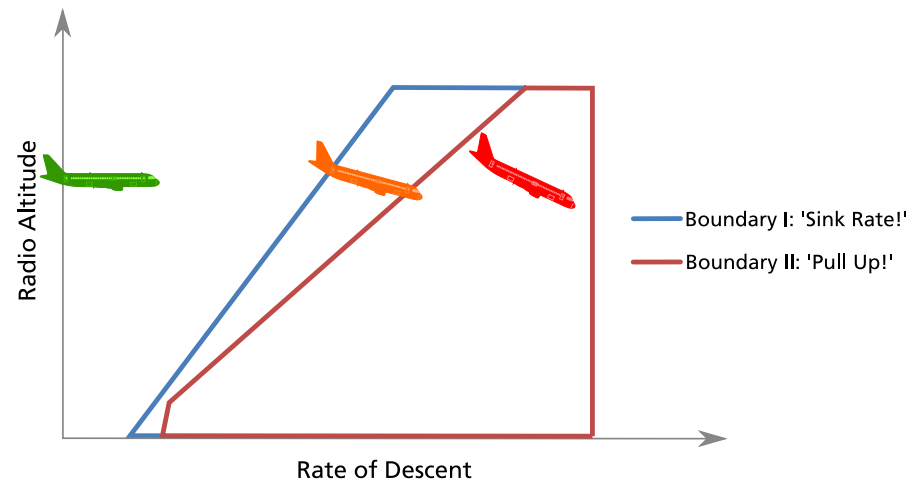

Fig. 2 Mode 1: Excessive Descent Rate.

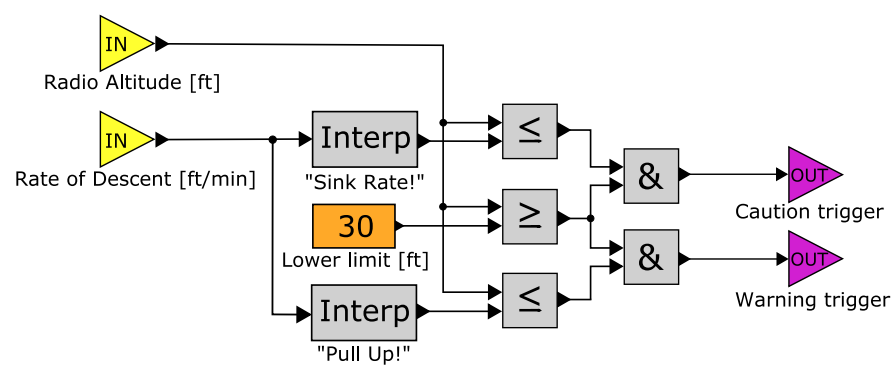

Fig. 3 Draft of the Scilab/Xcos diagram for Mode 1: Excessive Descent

Rate.

- Excessive Descent Rate: warnings for excessive descent rates for all phases of flight.

- Excessive Terrain Closure Rate: warnings to protect the aircraft from impacting the ground when terrain is rising rapidly with respect to the aircraft.

- Altitude Loss After Take-off: warnings when a significant altitude loss is detected after take-off or during a low altitude go around.

- Unsafe Terrain Clearance: warnings when there is no sufficient terrain clearance regarding the phase of the flight, aircraft configuration and speed.

- Excessive Deviation Below Glideslope: warnings when the aircraft descends below the glideslope.

Additionally, a TAWS may provide some enhanced functions, like the Terrain Awareness Display and Terrain Look Ahead Alerting, which rely on a terrain elevation database. They are included in the EGPWS by Honeywell which is installed in DLR's Airbus A320 Advanced Technologies Research Aircraft (ATRA). The enhanced functions relevant for this paper will be described in more detail in section IV.

The decision by DLR to develop an interpretation of the EGPWS - which is derived from the Pilot Manual - as a use case was due to the fact that the system has an immanent potential to be parallelized: the five basic modes and also the enhanced functions operate independently from each other. 


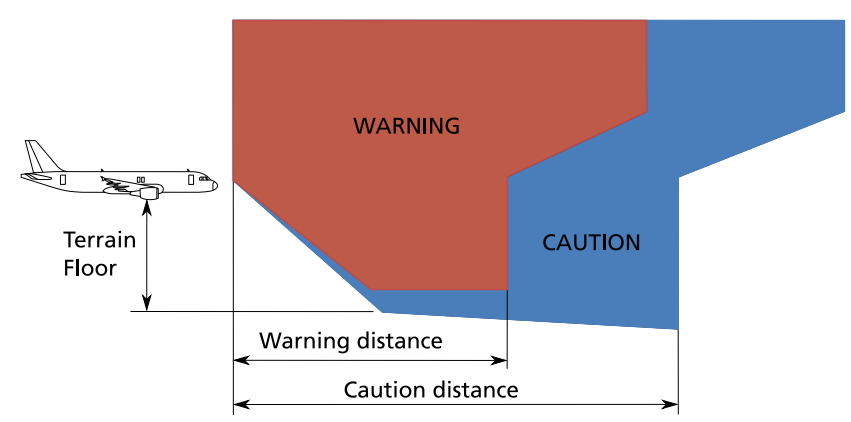

Fig. 4 Vertical geometry of the Terrain Look Ahead Alerting's caution and warning envelopes.

\section{DEVELOPMENT OF DLR's TAWS}

Following ARGO's model-based design workflow and utilizing the integrated toolchain, the basic modes of the TAWS have been developed using Scilab/Xcos. The algorithms for the conventional enhanced functions such as Terrain Awareness Display and Terrain Look Ahead Alerting are implemented within the model as Scilab scripts.

The Excessive Descent Rate mode that is depicted in Fig. 2 can be introduced as an example. An overview of its implementation in Scilab/Xcos is given in Fig. 3. The three aircraft have the same altitude of about $2000 \mathrm{ft}$, but different rates of descent, which is demonstrated by their position in the graph and their orientation. While the green aircraft is in a safe flight state, the orange one's rate of descent causes a warning. The red aircraft's descent rate, however, is too high considering its low altitude, requiring immediate action by the pilot. More details on this development effort can be found in [9].

\section{CONVENTIONAL AND NEW ENHANCED FUnCTIONS}

Of all the EGPWS functions available, two have been chosen to be realized for the ARGO use case: the Terrain Look Ahead Alerting and the Terrain Alerting Display. Other features like the Terrain or Runway Field Clearance Floor (TCF or RFCF, respectively) have been excluded since the estimated workload for their implementation was considered much higher than the payoff that is to be expected within the context of DLR's Air Vehicle Simulator (AVES). There, the new TAWS is going be integrated in the A320 cockpit.

The Terrain Look Ahead Alerting makes the pilot aware of potential conflicts with the terrain, based on the information that is stored in the EGPWS' terrain elevation database. The algorithm requires the aircraft's position, track, ground speed and altitude as input parameters. With these, two virtual envelopes representing several possible trajectories are projected forward of the aircraft and checked for collisions. These envelopes are called "ribbons" [10]. The bigger one, with a range equivalent to up to 60 seconds of flight, triggers a caution and the smaller one will result in a warning if a collision is found to occur in 30 seconds or less. Both ribbons start with a width of $1 / 4 \mathrm{~nm}$ and extend outwards laterally at an angle of $3^{\circ}$, while first running down, forward and finally up

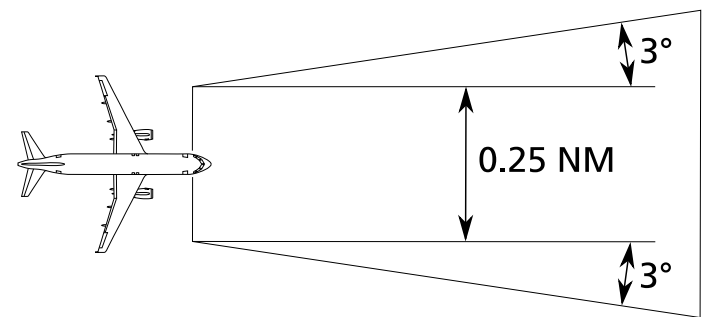

Fig. 5 Horizontal geometry of the Terrain Look Ahead Alerting's caution and warning envelopes.

higher than the aircraft's current altitude (see Fig. 4 and Fig. 5). As this shape is designed to yield several possibilities of the aircraft starting to sink or not being able to climb sufficiently, it may consequently result in nuisance warnings.

In order to avoid this, it was decided to replace the two envelopes by a predictor function that, in comparison, very exactly calculates the future position of the aircraft and checks it for terrain conflicts.

Honeywell's Terrain Alerting and Display in its original implementation features a terrain image that can be switched on and off on the Navigation Display. The visible geographic area is determined by the display range of the ND which can be set to $10,20,40,80,160$ or 320 nautical miles. The terrain itself is colored according to the threat it poses to the aircraft, whereas terrain lying at or above the aircraft's altitude is colored yellow to red and terrain below is colored green to black.

Conventionally, when a caution or warning alert is triggered by the Terrain Look Ahead Alerting, the critical area of the terrain image will be lit up in the ND [10]. A solid yellow corresponds to a caution and a bright red to a warning alert. As the ribbons cover a section of airspace not only in front of, but also well below the aircraft, even terrain that does not pose an immediate threat will be brought to the pilot's attention by the aural alert and the optical cue in the ND.

While this helps to maintain a safety clearance off the terrain, it leaves the final assessment of the situation to the pilot: is the alert due to an impending collision or to an undershooting of the required clearance? Is it better to climb or to pass the critical terrain by making a turn? If climbing, how much altitude should be gained and how fast? Alternatively, can the alert probably be regarded as a nuisance warning?

To decrease the pilot's cognitive workload that is caused by these questions, the authors propose the following enhancements to the PFD and the ND, all of which are activated only in case of a caution or a warning:

- Optical cues in the PFD's attitude indicators, visualizing the minimum Rate of Climb (ROC) and roll angles that are necessary to execute an evasive maneuver

- A red Flight Path Predictor (FPP) symbol, shaped like the aircraft symbol, showing the aircraft's position $60 \mathrm{~s}$ ahead in time in the ND's terrain image 


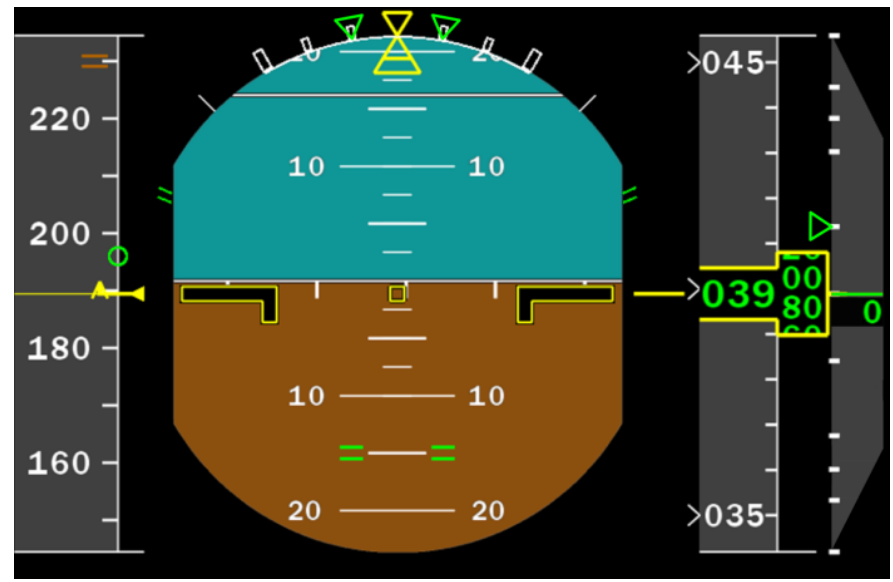

Fig. 6 Example for the bank angle and Rate of Climb target values presented as bugs in the PFD.

- One or two bright green symbols, shaped like the predictor symbol, showing suggestions for horizontal evasion maneuvers (left or right turn; if available) in the ND's terrain image

Depending on the situation, a straightforward vertical maneuver might be the most practical choice of action to avoid the terrain, as no changes to the course have to be made. However, with the optical cues available in an ND alone, no information can be given about the required ROC to stay clear of the obstacle. It was therefore decided to make use of the PFD's vertical speed indicator to close this gap. There are two conceivable means by which target values can be displayed: the first one is by using symbols called "bugs", and the second by coloring the safe and unsafe areas of the meter in green and red, respectively. The second method is already present in Terrain Collision Avoidance Systems (TCAS), where the direction and necessary extent of a vertical avoidance maneuver are conveyed to the pilot in the same way.

Without regard of which approach is chosen, a future effort should be to make this feature compatible to TCAS, so that the advisories from each system complement each other, rather than contradict or cancel each other out.

Considering performance, a horizontal maneuver may well be a more favorable option in certain cases. Again, one way to inform the pilot about the required action can be a bug on the attitude indicator, representing the minimum bank angle necessary to initiate a sufficient turn. Similar to the ROC cue on the vertical speed indicator, this can alternatively be a color coding in green and red. However, depending on the terrain ahead, both a left and a right turn may be feasible, resulting in two target values to be displayed. In any case, the ROC and the bank angle cues should be implemented coherently. See Fig. 6 and Fig. 7 for examples of the two approaches.

The aircraft's future position as shown by the Flight Path Predictor must be identified as precisely as possible, in order to allow for the absence of wide safety margins as they are present in the ribbons of Honeywell's Terrain Look Ahead Alerting. The authors plan to achieve this by utilizing DLR's Generic Aircraft Simulation (GAS), a simplified version of the A320 simulation model employed in AVES that uses linearized aerodynamic derivatives.

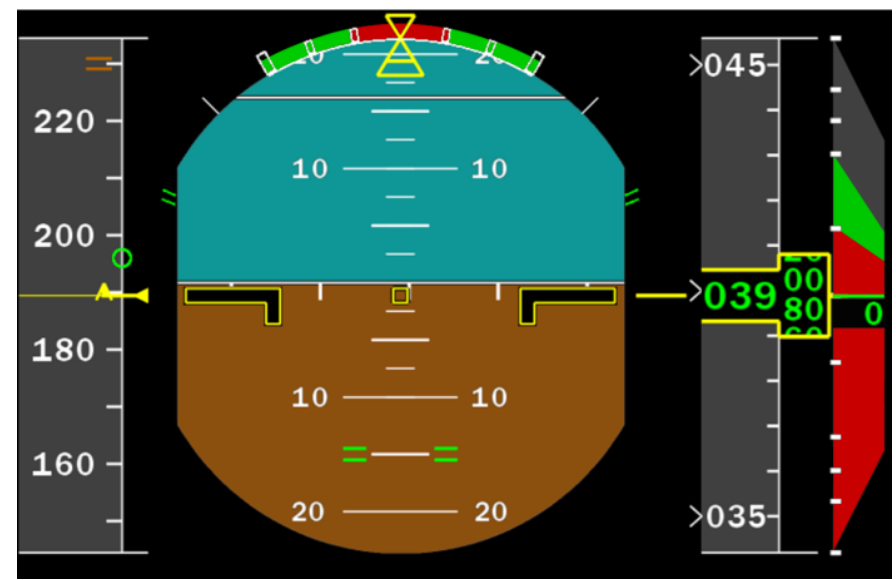

Fig. 7 Example for the bank angle and Rate of Climb target values presented as green areas in the PFD.

In forward flight, the GAS will accept the current control inputs, flight control settings and sensor data as parameters to calculate the position of the aircraft in 60 seconds, provided that the control input is left unchanged. For example, in horizontal steady flight, the predicted position will be straight ahead and at the same altitude as the aircraft. The GAS simulations will be executed continuously, making sure to keep the prediction up to date with changes in the control inputs.

Accurate predictions during a turn are harder to achieve, as there is no definite information about how long the pilot will continue to turn. An active flight plan may not suffice as a guarantee, but it can be the base to make an assumption for when the turn is complete. Therefore, as long as the aircraft follows the flight path designated by the Flight Management and Guidance Computer (FMGC), turns are assumed to end as soon as the aircraft is directed towards the next waypoint. The remainder of the predicted flight time is assumed to be spent without turns.

In case of angular deviations from the flight path however, these assumptions are not valid. In addition, the approach to extrapolate the current flight state for 60 seconds is much likely to lead to nuisance warnings, as the simulated turns will often continue for longer than the pilot desires. One example is a change of course of $60^{\circ}$ with the standard turn rate of $3^{\circ}$ per second. While this will take only 20 seconds in reality, the prediction would continue the turn for another 40 seconds, leading to a complete turnaround $\left(180^{\circ}\right)$. This is problematic as it causes the algorithm to consider terrain for the collision detection that the aircraft is currently passing by, and needs to be avoided.

Therefore, a different approach is necessary for outside the fight path or for free flight. The authors propose to have the algorithm assume that any turn in this domain ends after a change of course of $45^{\circ}$ - whereas the exact number is subject to research - and forward flight resumes. As the GAS is performing the simulations continuously, any conflicting terrain will still be recognized as the turn continues, thus covering the cases where a complete turnaround is indeed the pilot's intention. 


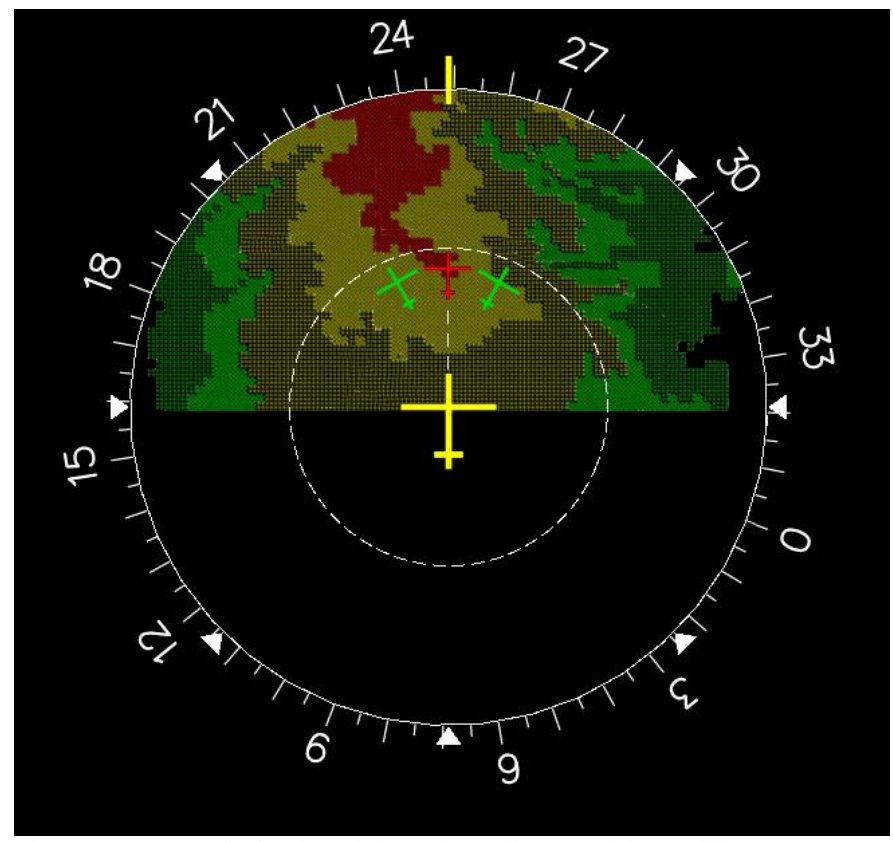

Fig. 8 Example for the Flight Path Predictor and the avoidance maneuver symbols in the Navigation Display.

If an impeding collision has been identified, it is visualized by displaying the FPP as a second, red aircraft symbol on the ND. It is located at the furthest point of the prediction, i.e. 60 seconds ahead, and remains visible until the obstacle is no longer a threat. Through the continuous simulations, the consequences for the flight path will be directly visible as movements of the FPP as soon as control changes (or other influences) are introduced.

In addition to the flight path prediction, the GAS will be used to evaluate avoidance maneuvers as well as to identify the target values for the above described PFD cues. The idea is to run three initial simulations: a left turn, a right turn and a strictly vertical evasion. The maximum bank angles for the turns are $10^{\circ}$ and $-10^{\circ}$ and all three simulated maneuvers feature a ROC of 1000 feet per minute. If all positions along these simulated 60 second flight paths are free of conflicts with the terrain (including a safety margin), they will be used as the source for the cues in the PFD and the bright green aircraft symbols in the ND that represent the horizontal avoidance maneuvers (Fig. 8).

If one of the turn simulations remains conflicted, it will be repeated, first with $15^{\circ}$, then $20^{\circ}$ of maximum bank angle and so forth, if necessary. However, if the vertical evasion is found to be insufficient, not only will itself be repeated with an ROC of 2000 feet per minute (and again with 3000, if required), but the turning maneuvers will also be simulated anew, starting at $+/-10^{\circ}$ of bank angle and utilizing the new climb rate. This is necessary to keep the displayed target values for ROC and bank angle consistent with each other and the conducted simulations.

1 A turn maneuver in the simulation will consist of two rolling movements: one to achieve the maximum bank angle, and the second, following suit, to return to a bank angle of $0^{\circ}$.
For example, a mountain range straight ahead of the aircraft is detected by the algorithm as a terrain conflict. The first run of the three simulations does not bring up any sufficient maneuvers. All three simulations are repeated with an ROC of 2000 feet per minute, although making more narrow turns with a higher bank angle instead may solve the problem as well. However, this would mean that, along with the sufficient bank angle, the - in itself insufficient - climb rate of 1000 feet per minute was displayed in the PFD, which is considered counterproductive by the authors.

It should be mentioned that air density and temperature, as well as the current aircraft mass are parameters of the GAS, so that performance issues are not ignored or left up to assumptions. Furthermore, the aircraft's stall speed and maximum load factor are considered to exclude maneuvers that would lead to unsafe flight states. In the case that the desired terrain clearance cannot be achieved due to one or several of these restrictions, the last resort is to minimize the safety margin and repeat the last simulation so that an avoidance maneuver may still be found.

Running the path evaluating simulations will continue until at least one sufficient evasion maneuver is found. After that, the thus identified maneuver(s) will continue to be simulated along with the flight path prediction, in order to allow for immediate adaption to new circumstances, like more terrain that had not been in range for the detection before. This way, the pilot will be given as much time as possible to react to new conflicts along the avoidance path. See Fig. 8 again as an example: if the pilot chooses the left maneuver, he will still be heading towards conflicting terrain. But as the simulation of the same maneuver continues to predict the next 60 seconds, the conflict will be detected and the maneuver will be adapted.

Once the obstacle detection finds that there are no conflicts left along the predicted flight path, the simulation of avoidance maneuvers will cease and the FPP symbol will turn from red to white. 5 seconds later, it will disappear completely to avoid unnecessary display cluttering.

The Scilab/Xcos model of the GAS, running with a step size of 0.1 seconds to a simulated time of 17 seconds, was experienced to be considerably slower than real time during model-in-the-loop tests with the basic TAWS. Therefore, it is not unlikely that the above described approach will overstrain the capabilities of the target hardware. However, the optimization and parallelization of the software that is promised by the ARGO toolchain, together with the performance boost by the multicore hardware, may be able to solve this shortcoming. If this is not the case, the hardware requirements will have to be lowered by increasing the step size in as many iterations as necessary until the GAS can be run without frame drops. Of course, the achievable precision will then have to be evaluated.

\section{THE DECISION FOR PFD AND ND}

Since the introduction of Honeywell's EGPWS in 1996, several other TAWS have been developed. Some of them (like the EGPWS integrated with BendixKing's AeroVue [11] or Rockwell Collins' Helisure Helicopter Synthetic Vision System [12]) offer a synthetic view from the cockpit and are 
therefore called Synthetic Vision Systems (SVS). They are usually combined with an arrangement of altitude, airspeed and track meters as well as attitude indicators, enabling them to act as replacements - if their certification allows for it - for the conventional PFD. Given that a lack of situational awareness was found to be the reason for most CFIT accidents, presenting the pilot with an artificial and enhanced view of the upcoming terrain seems to be the obvious choice for further improving the avoidance capabilities.

However, Andre and Wickens suggest otherwise in [13]. They claim that sometimes "users want what's not best for them", hinting at how 3D is not superior to 2D in every aspect or for every task. For example, the location of objects along lines of sight into the display's viewing plane is ambiguous [14].

Also, space in a 3D view is nonlinearly compressed, resulting in the distortion of distances and angles [15]. Picking up on these findings, St. John concluded that 3D displays are to be preferred for tasks that demand an intuitive understanding of the shape of objects or scenes, whereas 2D displays are better for precise judgment of relative positions [16].

In the case of TAWS, it is possible to argue for either: on the one hand, understanding the shape of the terrain in general may be more important for evasive maneuvers than precise judgment of the distance to the conflicting terrain. Consequently, St. John arrived at the conclusion that avoidance tasks are best performed with a 3D display [16]. On the other hand, however, a two-dimensional top view of the terrain may well have advantages of its own.

As the approach of upgrading the PFD to a threedimensional SVS display has been used by various manufacturers before, the authors considered it more rewarding to explore the potential of a further enhanced 2D Navigational Display. This decision is also supported by the fact that a terrain image in an SVS requires rendering of the threedimensional terrain, which is a necessity that can be avoided with 2D displays.

Another possible advantage is that a color-coded terrain image with a predictor symbol will likely not only help the pilot to meter the distance to impact more precisely and thus assess the criticality of a potential collision, but will also allow him to plan ahead further than he can directly see. In contrast, due to the virtual line of sight in an SVS, the presented terrain may obstruct the view of more obstacles in a 3D display.

Finally, utilizing a conventional EFIS in the described way rather than an integrated SVS allows for a compromise between two extremes: offering complete situational awareness on the one hand and merely advising the pilot how to evade on the other hand. While the former has the advantage that the pilot can make a thought-out decision based on a full understanding of the whole situation, this process is likely to be time-consuming [17]. Evasion commands in the way of a Flight Director are easy to act on quickly, but do not keep the pilot informed about the reasons for the maneuver. Therefore, the aforementioned approach was chosen.

\section{CONCLUSION}

In this paper, the use of parallelized applications on multicore platforms for avionics, especially their potential benefits, has been discussed. The focus was placed on an TAWS that is developed as a use case for the EU-project ARGO.

After introducing the project and giving insight into the model-based development with Scilab/Xcos, the conventional features of Honeywell's EGPWS have been described, followed by an explanation of the new enhanced features currently in development at DLR. Finally, the decision to add functionality to the conventional Electronic Flight Instrument System, rather than employing a Synthetic Vision System, was discussed.

The next steps include the implementation of the described approach with Scilab/Xcos. Parallel $\mathrm{C}$ code will be automatically generated by the toolchain and will then have to be applied to the target hardware designated by the ARGO consortium. Then the final step will be to integrate it into the simulation infrastructure of AVES in order to conduct pilot-inthe-loop validation.

\section{ACKNOWLEDGMENT}

This work is funded by the European Union under the Horizon 2020 Research and Innovation Program, Grant Agreement ICT-2015-688131, project "WCET-Aware Parallelization of Model-Based Applications for Heterogeneous Parallel Systems (ARGO)" (http://www.argoproject.eu/).

\section{REFERENCES}

[1] Nowotsch, J. and M. Paulitsch. 2012. "Leveraging multi-core computing architectures in avionics". In 9th European Dependable Computing Conference (EDCC), Sibiu, Romania.

[2] Karray, H., M. Paulitsch, B. Koppenhöfer, and D. Geiger. 2013. "Design and implementation of a degraded vision landing aid application on a multicore processor architecture for safety-critical application". In 16th International Symposium on Object/Component/Service-Oriented RealTime Distributed Computing (ISORC), Paderborn, Germany.

[3] Koppenhöfer, B., and D. Geiger. EMC2 Use Case:Hybrid Avionics Integrated Architecture Demonstrator. HiPEAC, Workshop EMC 2015.

[4] Agrou, H., P. Sainrat, M. Gatti, and P. Toillon. 2012. "Mastering the behavior of multi-core systems to match avionics requirements". In AIAA 31st Digital Avionics Systems Conference (DASC), Williamsburg, VA.

[5] Sander, O., F. Bapp, L. Dieudonne, T. Sandmann, and J. Becker. 2017. "The promised future of multi-core processors in avionics systems". CEAS Aeronautical Journal vol.8, no. 1, pp. 143-155.

[6] Campbell, S.L., J.P. Chancelier, and R. Nikoukhah. 2010. Modeling and Simulation in Scilab/Scicos with ScicosLab 4.4. Springer New York.

[7] Derrien, S., I. Puaut, P. Alefragis, M. Bednara, H. Bucher, C. David, Y. Debray, U. Durak, I. Fassi, C. Ferdinand und D. Hardy, „WCET-aware parallelization of model-based applications for multicores: The ARGO approach," in Design, Automotion \& Test in Europe Conference \& Exhibiton (DATE), Lausanne, Switzerland, 2017.

[8] Federal Aviation Administration, Advisory Circular 23-18, Installation of Terrain Awareness and Warning Systems (TAWS) Approved for Part 23 Airplanes, Washington DC: U.S. G.P.O., 2000. 
[9] Durak, U., D. Müller, J. Becker, N. Voros, P. Alefragis, T. Stripf, P. Agnel, G. Rauwerda und K. Sunesen, „Model-based development of Enhanced Ground Proximity Warning System for hetereogeneous multicore architectures," in Tagungsband ASIM 2016, Dresden, Germany, 2016.

[10] Honeywell International Inc., MK V and MK VII Pilot's Guide, 0604241-0000, Rev. H, 2011.

[11] BendixKing, AeroVue ${ }^{\mathrm{TM}}$ Integrated Flight Deck for the Beechcraft King Air B200, Albuquerque, 2017

[12] Rockwell Collins, HeliSure ${ }^{\mathrm{TM}}$ Helicopter Synthetic Vision System (HSVS), Blagnac Cedex, France, 2013.

[13] Andre, A. und C. Wickens, „When users want what's not best for them,“ Ergonomics in Design, pp. 10-14, October 1995.

[14] Boyer, F. M. Campbell, D. Merwin und C. Wickens, „Three-dimensional displays for terrain and weather awareness in the national airspace system," in Proceedings of the Human Factors and Ergonomics Society 39th Annual Meeting, Santa Monica, CA, 1995.

[15] Gillam, B., „The perception of spatial layout from static optical information," in Perception of space and motion, 2. Hrsg., W. a. R. S. Epstein, Hrsg., San Diego, CA, 1995, pp. 23-67.

[16] St.John, M., M. Cowen, H. Smallman und H. Oonk, ,The Use of 2D and 3D Displays for Shape-Understanding versus Relative-Position Tasks,“ Human Factors, Nr. Vol. 43, No. 1, pp. 79-98, 2001.

[17] Mulder, M., Cybernetics of tunnel-in-the-sky displays, Delft, 1999. 